\title{
Intrarenal Resistance Index as a Prognostic Parameter in Patients with Liver Cirrhosis Compared with Other Hepatic Scoring Systems
}

\author{
M. Götzberger ${ }^{\mathrm{a}} \quad$ J. Singer ${ }^{\mathrm{a}} \quad$ C. Kaiser ${ }^{\mathrm{b}} \quad$ V. Gülberg ${ }^{\mathrm{a}}$

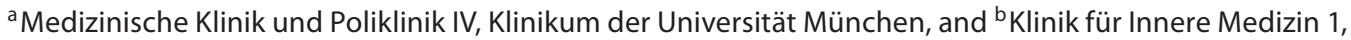 \\ Klinikum Dritter Orden, München, Germany
}

\section{Key Words}

Liver cirrhosis $\cdot$ Resistance index $\cdot$ Hepatorenal syndrome $\cdot$ MELD score

\begin{abstract}
Background and Aims: Patients with advanced liver cirrhosis who develop renal dysfunction have a poor prognosis. Elevated intrarenal resistance indices (RIs) due to renal vascular constriction have been described before in cirrhotic patients. In the current study, we prospectively investigated the course of intrarenal Rls and compared their prognostic impact with those of the Model for End-Stage Liver Disease (MELD) and the Child-Pugh scores. Methods: Sixty-three patients with liver cirrhosis underwent a baseline visit which included a sonographic examination and laboratory tests. Forty-four patients were prospectively monitored. The end points were death or survival at the day of the follow-up visit. Results: In 28 patients, a follow-up visit was performed after $22 \pm 8$ months (group 1). Sixteen patients died during follow-up after $12 \pm 8$ months (group 2). Group 2 patients showed a significantly higher baseline RI $(0.76 \pm 0.05)$ than group 1 patients $(\mathrm{RI}=0.72 \pm 0.06 ; \mathrm{p}<0.05)$. As shown by receiver operating characteristic analysis, the $\mathrm{Rl}$ and the MELD score achieved similar sensitivity and specificity [area under the curve (AUC): $0.722 ; 95 \%$ confidence interval (95\% Cl): $0.575-0.873$ vs. AUC: $0.724 ; 95 \% \mathrm{Cl}: 0.575-0.873, z=0.029$,
\end{abstract}

n.s.] in predicting survival and were superior to the ChildPugh score (AUC: 0.677; 96\% Cl: 0.518-0.837). Conclusion: The Rl is not inferior in sensitivity and specificity to the MELD score. Cirrhotic patients with elevated Rls have impaired short- and long-term survival. The RI may help identify highrisk patients that require special therapeutic care.

Copyright $\odot 2012$ S. Karger AG, Basel

\section{Introduction}

Advanced liver cirrhosis is associated with a poor clinical outcome [1]. Therefore, assessment of prognosis is important in the management of these patients. The Child-Pugh score has long been the most widely used specific scoring system in liver disease [2, 3]. In 2002, the Model for End-Stage Liver Disease (MELD) was introduced for patients undergoing transjugular intrahepatic portosystemic shunt. It is currently used to predict survival in patients awaiting liver transplantation $[3,4]$. The MELD seems to be superior to the Child-Pugh score in prioritizing potential liver recipients according to mortality risk [5]. However, it is only based on three laboratory variables, and thus does not take into account all prognostic factors that will impact on the survival of cirrhotic patients, notably complications due to portal hypertension [4]. There is still a need for improvement of

\section{KARGER}

Fax +4161306 1234

E-Mail karger@karger.ch

www.karger.com (c) 2012 S. Karger AG, Basel

0012-2823/12/0864-0349\$38.00/0

Accessible online at:

www.karger.com/dig
Manuela Götzberger, MD

Medizinische Klinik und Poliklinik IV, Klinikum der Universität München Ziemssenstrasse 1

DE-80336 München (Germany)

E-Mail manuela.goetzberger@med.uni-muenchen.de 


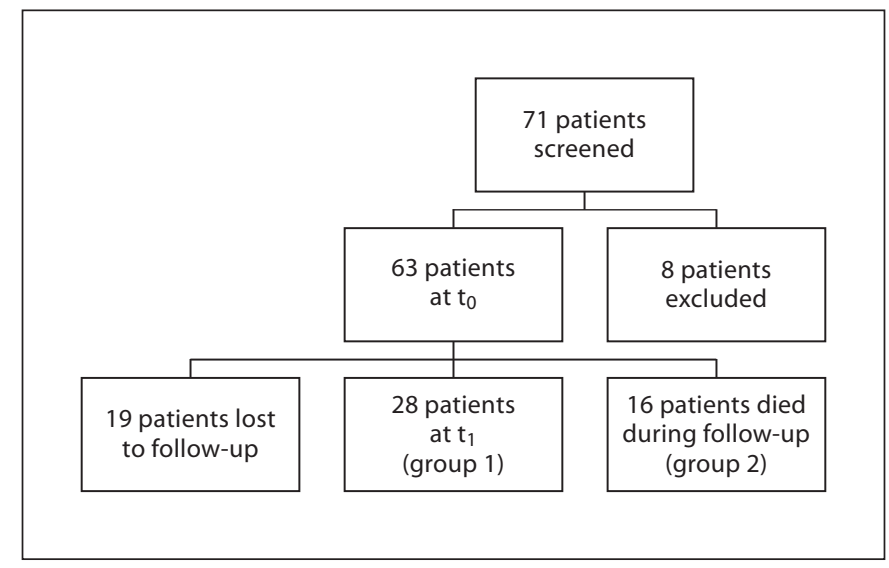

Fig. 1. Study design: 44 patients were prospectively monitored and divided in two groups. Patients of group 1 were also seen at $t_{1}$. Patients of group 2 died during the follow-up.

prognostic markers that could be easily integrated into the clinical management of these patients.

Patients with liver cirrhosis frequently develop renal dysfunction. The hepatorenal syndrome (HRS), the most serious renal complication, is associated with an extremely short survival time [6]. The HRS is characterized by renal arterial vasoconstriction, which may precede clinically manifest renal dysfunction. The intrarenal resistance index (RI) is the most frequently used parameter to assess intrarenal resistance and is calculated based on Doppler sonographic intrarenal measurements. It is routinely used to diagnose transplant rejection or renal artery stenosis $[7,8]$. Increased intrarenal RIs in patients with liver cirrhosis, especially in the decompensated stage, have been described before as compared to healthy controls [9-13]. Cirrhotic patients with elevated intrarenal RIs tend to develop the HRS, leading to a poor prognosis. In the current study, we prospectively investigated the course of intrarenal RIs in patients with liver cirrhosis and compared its prognostic impact with those of the MELD and the Child-Pugh scores.

\section{Subjects and Methods}

\section{Study Subjects}

Adult patients ( $\geq 18$ years old) with known liver cirrhosis, admitted to our outpatient clinic for surveillance of cirrhosis, were screened and enrolled in the study. Patients with suspected or overt malignant diseases, a history of insulin-dependent diabetes mellitus or any other nephropathy as well as patients with ongoing HRS, gastrointestinal bleeding, spontaneous bacterial perito- nitis or other acute infections at the time of the baseline visit $\left(\mathrm{t}_{0}\right)$ were excluded. A follow-up of at least 6 months was required for inclusion in the study. Informed consent was obtained from all patients before their inclusion.

\section{Study Design}

At the time of the baseline visit, all patients underwent sonographic examination of the liver and kidneys, including Doppler evaluation of intrarenal resistance.

Patients were fasting $4 \mathrm{~h}$ prior to examination. For duplex Doppler ultrasound examination, a $3.5-\mathrm{MHz}$ convex transducer was used (Hitachi EUB-8500 and Siemens Sonoline Elegra). Sonographic examinations were performed by two experienced investigators, thus precluding calculation of interobserver variation. The intraobserver coefficient of variation ranged from 3.2 to $3.4 \%$ (M.G.) and from 3.0 to $3.7 \%$ (C.K.). The RI was calculated with the following formula: RI = (peak systolic velocity - end diastolic velocity)/peak systolic velocity. Intrarenal resistance was measured on interlobar arteries three times in different regions of each kidney. Subsequently, a mean RI was calculated for each patient (mean of both kidneys).

Laboratory tests for liver and renal function were performed on each patient and clinical parameters, such as blood pressure, heart rate and age, were measured. Hepatic encephalopathy was clinically assessed and classified according to the West Haven scale (0-4) [14]. Ascites was graded as absent, mild to moderate or severe based on sonography.

Patients were prospectively monitored. The end points were death or survival at the day of the follow-up visit $\left(t_{1}\right)$. On the follow-up visit, the same parameters were investigated as on the baseline visit $\left(\mathrm{t}_{0}\right)$.

\section{Statistical Analysis}

All data were expressed as means \pm standard deviations. Statistical analysis was performed using SPSS. Differences between groups were analyzed by Student's t test; $\mathrm{p}<0.05$ was considered significant. To discriminate the predictive value of the parameters, a receiver operating characteristic (ROC) curve was established by standard procedures [15]. Accordingly, a z-value was calculated for comparing the MELD score and the RI. Assuming a two-tailed probability, a $z$-value $>1.96$ was taken as evidence that ROC areas were different [16]. Survival curves were evaluated and compared using the Kaplan-Meier method and the log-rank test; $\mathrm{p}<0.05$ was considered significant.

\section{Results}

Seventy-one consecutive patients with liver cirrhosis were screened. Eight patients were excluded because of diabetes or other nephropathy in 6 cases and malignant disease in 2 cases. Sixty-three patients presented at the baseline visit $\left(\mathrm{t}_{0}\right)$, but 19 patients were lost to follow-up. Finally, 44 patients were enrolled in the follow-up study. Twenty-eight patients underwent a follow-up visit $\left(\mathrm{t}_{1}\right)$ after $22 \pm 8$ months (group 1). Sixteen patients (36\%) died after $12 \pm 8$ months (group 2) (fig. 1). Causes of death are 
Table 1. Causes of death during follow-up in group 2 patients with liver cirrhosis $(\mathrm{n}=16)$

\begin{tabular}{ll}
\hline & Patients, $\mathrm{n}$ \\
\hline HRS & 5 \\
Liver failure & 3 \\
Upper gastrointestinal bleeding & 1 \\
Rectum carcinoma & 1 \\
Cerebral bleeding & 1 \\
Sepsis & 1 \\
Unknown & 4 \\
\hline
\end{tabular}

Table 2. Baseline characteristics of both groups at $t_{0}$

\begin{tabular}{lcc}
\hline & Group 1 (n=28) & Group 2(n=16) \\
\hline Etiology, $\mathrm{n}$ & & \\
Alcohol abuse & $17(60 \%)$ & $12(75 \%)$ \\
Chronic hepatitis B or C & $7(25 \%)$ & $3(19 \%)$ \\
Wilson's disease & $1(4 \%)$ & 0 \\
Autoimmune hepatitis & $1(4 \%)$ & 0 \\
Primary biliary cirrhosis & 0 & $1(6 \%)$ \\
Cryptogenic & $2(7 \%)$ & 0 \\
Biochemical parameters & & \\
Serum bilirubin, mg/dl & $2.7 \pm 3.0$ & $5.8 \pm 10.0$ \\
Serum albumin, g/dl & $3.5 \pm 0.8$ & $3.1 \pm 0.8$ \\
Prothrombin index, \% & $72 \pm 17$ & $59 \pm 16$ \\
Serum creatinine, mg/dl & $0.9 \pm 0.3$ & $1.0 \pm 0.5$ \\
Clinical parameters & & \\
Ascites & $12(43 \%)$ & $12(75 \%)$ \\
Encephalopathy & $13(46 \%)$ & $6(37 \%)$ \\
$\quad$ Grade I & $1(4 \%)$ & $4(25 \%)$ \\
$\quad$ Grade II & $12(43 \%)$ & $12(75 \%)$ \\
Blood pressure, systolic & $115 \pm 18$ & $117 \pm 13$ \\
Blood pressure, diastolic & $72 \pm 13$ & $73 \pm 7$ \\
Heart rate & $82 \pm 8$ & $77 \pm 10$ \\
BMI & $26.7 \pm 3.5$ & $25.3 \pm 5.1$ \\
Body weight, kg & $80.8 \pm 11.2$ & $74.6 \pm 13.7$ \\
Portal vein flow & $16 \pm 5$ & $16 \pm 6$ \\
Portal vein reversal, $\mathrm{n}$ & $4(14 \%)$ & $4(25 \%)$ \\
\hline
\end{tabular}

shown in table 1 and patients' baseline characteristics of both groups, in table 2. Group 2 patients showed a baseline RI of $0.76 \pm 0.05$ at $t_{0}$, which is significantly higher than the baseline RI of group $1(\mathrm{RI}=0.72 \pm 0.06, \mathrm{p}<$ $0.05)$. The RI was the only documented parameter that differed significantly between the two groups at $\mathrm{t}_{0}$ (table 3). In ROC analysis, the RI and the MELD score showed similar sensitivity and specificity [area under the curve (AUC): 0.722; 95\% confidence interval (95\% CI):

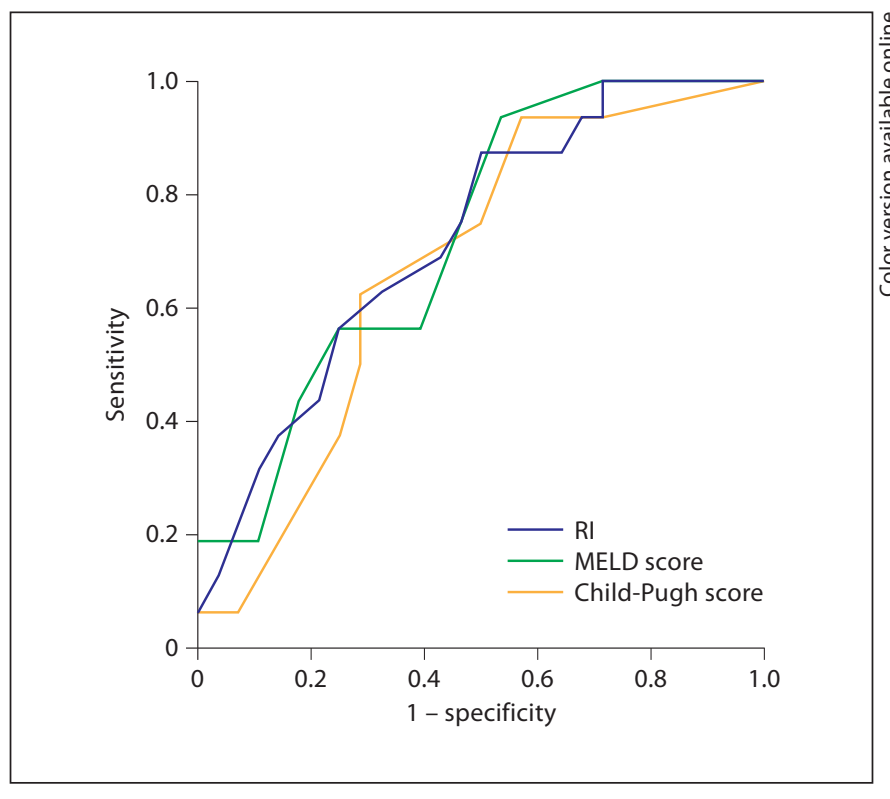

Fig. 2. ROC curve for the MELD score, Child-Pugh score and RI. The ROC curve for the MELD score and RI value showed similar performances.

$0.575-0.873$ vs. AUC: $0.724 ; 95 \%$ CI: $0.575-0.873, z=$ 0.029 , n.s.] in predicting survival and were superior to the Child-Pugh score (AUC: 0.677; 95\% CI: 0.518-0.837) (fig. 2). According to the best accuracy derived from ROC analysis, we chose a cut-off of 0.74 to compare survival among patients (sensitivity: 62.5\%; specificity: 68\%). Kaplan-Meier survival curves showed a significant difference in survival between patients with RI $>0.74$ and RI $\leq 0.74$ in the short-term as well as the long-term course ( $\mathrm{p}=0.037$, log-rank test) (fig. 3 ).

On the basis of previous studies, an RI $>0.70$ was considered elevated and thus indicative of renal vasoconstriction $[17,18]$. In group 2 , only 2 patients had RIs $<0.70$ at $\mathrm{t}_{0}$. One of these patients died of sepsis due to pneumonia. The cause of death of the second patient with a normal RI at $\mathrm{t}_{0}$ is unknown. All other patients $(88 \%)$ of group 2 had RIs $\geq 0.72$ at $t_{0}$ compared to only $50 \%$ of patients in group 1. Among the patients who died, the highest RIs $(0.78 \pm 0.04)$ were found in patients with the HRS.

In group 1 , the results at $t_{0}$ and $t_{1}$ revealed a stable course of the liver disease. Some patients' clinical condition even improved. At $t_{0}, 12$ patients presented with ascites, but only 7 patients did so at $t_{1}$. Consistent with the clinical results, patients showed a slight improvement in their Child-Pugh score, RI and MELD score (table 4). A subgroup of 3 patients showed impairment in serum cre- 


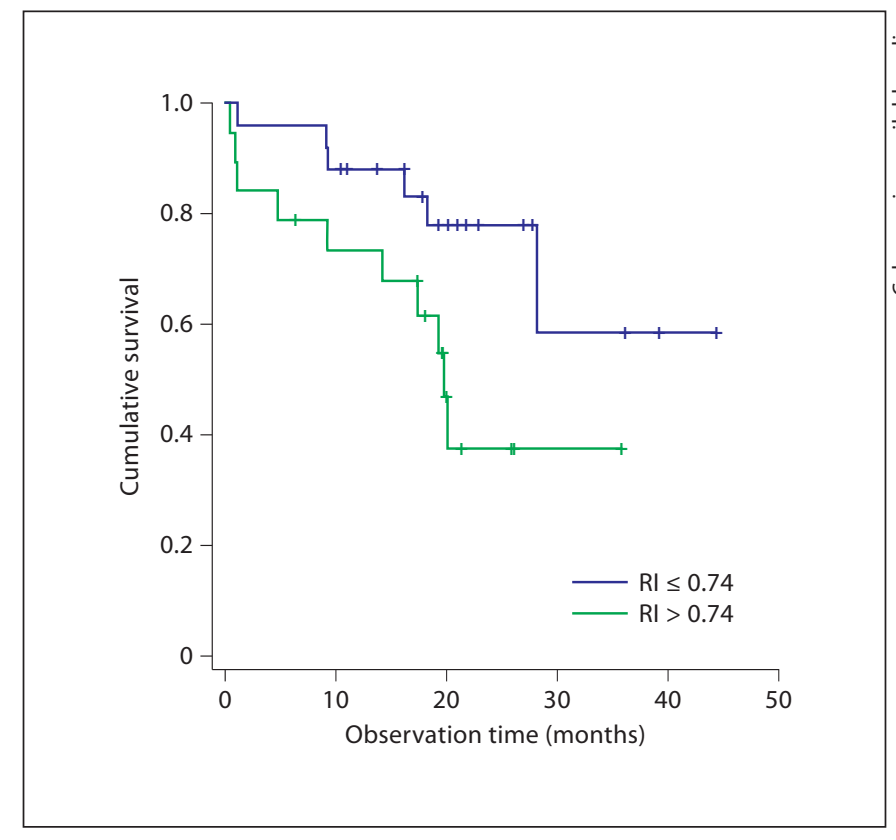

Fig. 3. Kaplan-Meier survival curves according to RI demonstrated a significantly better outcome in patients with RI $\leq 0.74(\mathrm{p}=$ 0.037, log-rank test).

atinine during the follow-up. Two patients with serum creatinine $>2 \mathrm{mg} / \mathrm{dl}$ at $\mathrm{t}_{1}$ had elevated RIs already at $\mathrm{t}_{0}$ ( $\mathrm{RI}=0.78$ and 0.77 , respectively). The patient with $\mathrm{RI}=$ 0.77 at $\mathrm{t}_{0}$ fulfilled the criteria of HRS at $\mathrm{t}_{1}$. The third patient demonstrated a drastically increased RI from 0.65 to 0.79 whereas serum creatinine was only slightly increased to $1.2 \mathrm{mg} / \mathrm{dl}$ at $\mathrm{t}_{1}$.

\section{Discussion}

The current study is the first to show the similar sensitivity and specificity of the intrarenal RI and the MELD score in assessing survival in patients with liver cirrhosis. Both parameters were superior to the Child-Pugh score in ROC analysis. In addition, we could confirm the findings of previous studies showing that cirrhotic patients with elevated RIs have impaired short- and long-term survival and are at higher risk of developing the HRS. Kaplan-Meier survival curves demonstrated a significantly better outcome in patients with RI $\leq 0.74$.

Liver cirrhosis is characterized by complex changes in systemic hemodynamics. Especially renal dysfunction frequently complicates the clinical course of this disease. Doppler ultrasound measurement of intrarenal resis-
Table 3. Parameters at $t_{0}$ of group 1 (alive at the end of follow-up) and group 2 (dead at the end of follow-up)

\begin{tabular}{lccc}
\hline & Group 1 $(\mathrm{n}=28)$ & Group 2 $(\mathrm{n}=16)$ & $\mathrm{p}$ \\
\hline & $57 \pm 10$ & $62 \pm 9$ & $\mathrm{n} . \mathrm{s}$. \\
Age, years & $21 / 7$ & $9 / 7$ & \\
RI & $0.72 \pm 0.06$ & $0.76 \pm 0.05$ & $<0.05$ \\
cGFR, ml/min & $91.1 \pm 25.0$ & $81.2 \pm 33.8$ & n.s. \\
Serum creatinine, mg/dl & $0.9 \pm 0.3$ & $1.0 \pm 0.5$ & n.s. \\
MELD score & $12 \pm 4$ & $16 \pm 6$ & n.s. \\
Child-Pugh score & $7.7 \pm 2.4$ & $9.2 \pm 2.0$ & n.s. \\
& & &
\end{tabular}

cGFR = Calculated glomerular filtration rate estimated according to the Modification of Diet in Renal Disease (MDRD) formula.

Table 4. Comparison of parameters in patients of group 1 at $t_{0}$ and $t_{1}$

\begin{tabular}{lccc}
\hline Group 1 $(\mathrm{n}=28)$ & $\mathrm{t}_{0}$ & $\mathrm{t}_{1}$ & $\mathrm{p}$ \\
\hline Age, years & $57 \pm 10$ & $59 \pm 10$ & n.s. \\
RI & $0.72 \pm 0.06$ & $0.69 \pm 0.06$ & $<0.03$ \\
GFR, ml/min & $91.1 \pm 25.0$ & $83 \pm 24$ & n.s. \\
Serum creatinine, mg/dl & $0.9 \pm 0.3$ & $1.0 \pm 0.4$ & n.s. \\
MELD score & $12 \pm 4$ & $11 \pm 4$ & n.s. \\
Child-Pugh score & $7.7 \pm 2.4$ & $6.6 \pm 2.0$ & $<0.02$ \\
\hline
\end{tabular}

tance can estimate renal blood flow and correlates with portal pressure [19]. Some studies have shown that intrarenal RIs are significantly increased in cirrhotic patients compared with healthy controls and are even higher in patients with ascites than in patients without ascites $[9$, 10]. Patients with decompensated cirrhosis but normal serum creatinine can already present with elevated RIs [12]. So far, two teams performed follow-up investigations of cirrhotic patients after measuring renal resistance. Maroto et al. [20] investigated 32 cirrhotic with ascites. The subgroup of 17 cirrhotic patients with renal failure showed elevated RIs of $0.74 \pm 0.01$. Follow-up revealed the RI as an indicator of impaired survival in the univariate analysis. Platt et al. [18] measured intrarenal resistance in 180 cirrhotic patients without kidney dysfunction. During the follow-up, the HRS developed in $26 \%(n=20)$ of patients with an initial RI $>0.70$, but only in $1 \%$ of those with normal RIs. The mean initial RI in patients who subsequently developed the HRS was 0.77 \pm 0.05 . Our findings are in agreement with previous results showing a mean RI of $0.78 \pm 0.04$ in the 5 patients 
of group 2 who died due to the HRS. In the present study, a further RI was obtained at $t_{1}$. The patients who were still alive at $t_{1}$ exhibited a stable course of their liver disease, possibly due to close clinical management. These results confirm that RIs and renal hemodynamics can improve with time. However, in a subgroup of 3 patients, impaired serum creatinine was found during the follow-up. Two patients with considerable worsening already had elevated RIs at $t_{0}$, comparable to those of patients of group 1 . This supports the assumption that an elevated RI is an earlier indicator of development of renal dysfunction followed by a rise in serum creatinine with time.

RIs $>0.70$ were defined as elevated in a number of studies $[17,18]$. Our data reveal that most of the high-risk patients had RIs $\geq 0.72$. The optimal cut-off level for elevated RIs should be validated on a larger number of subjects. Two patients in group 2 (12\%) showed normal RIs at baseline, which would have led to false-negative results in these cases. One of these patients died due to pneumonia during follow-up. The cause of death of the other patient is unknown. Apart from these aspects, other possible limitations of our results are important to consider: our study was designed as a single-center study and did not have an external validation group to further confirm the results.
In conclusion, we demonstrated for the first time that the RI is not inferior in sensitivity and specificity to the MELD score. At the present time, the MELD score is mainly used in the transplantation setting [5]. It is based on easily measured variables (prothrombin time, bilirubin and creatinine). Serum creatinine is an indicator of impaired renal function; however, it has disadvantages as it depends on muscle mass and physical activity. Therefore, renal function based on serum creatinine can be overestimated in patients with advanced cirrhosis [22]. Thus, it is still necessary to develop improved prognostic markers feasible in daily practice. Our study confirms that the RI, based on sonographic measurements of intrarenal resistance, is an effective, noninvasive, economical functional test that provides useful information for the prognosis and management of cirrhotic patients. Elevated RIs may even disclose progress of the liver disease before changes in laboratory results. Therefore, the RI may help identify a subgroup of high-risk patients with a poor prognosis that require special therapeutic care. According to clinical practice guidelines, cirrhotic patients should be entered into surveillance programs and undergo ultrasound examination every 6-12 months [23]. Hence, our data suggest to include the measurement of renal resistance in these surveillance programs.

\section{References}

1 Bell H, Jahnsen J, Kittang E, Raknerud N, Sandvik L: Long-term prognosis of patients with alcoholic liver cirrhosis: a 15-year follow-up study of 100 Norwegian patients admitted to one unit. Scand J Gastroenterol 2004;9:858-863.

$\checkmark 2$ Pugh RN, Murray-Lyon IM, Dawson JL, Pietroni MC, Williams R: Transection of the oesophagus for bleeding oesophageal varices. Br J Surg 1973;60:646-649.

-3 Freire P, Romaozinho JM, Amaro P, Ferreira M, Sofia C: Prognostic scores in cirrhotic patients admitted to a gastroenterology intensive care unit. Rev Esp Enferm Dig 2011;103: 177-183.

-4 Rowe I, Neuberger J: Prognostic models in cirrhosis: an aid but not a replacement for clinical judgement. Liver Intl 2007;27:595597.

5 Wiesner R, Edwards E, Freeman R, Harper A, Kim R, Kamath P, Kremers W, Lake J, Howard T, Merion RM, Wolfe RA, Krom R, the United Network For Organ Sharing Liver Disease Severity Score Committee: Model for End-Stage Liver Disease (MELD) and allocation of donor livers. Gastroenterology 2003;124:91-96.

Intrarenal Resistance Index in Patients with Liver Cirrhosis
-6 Ginès A, Escorsell A, Ginès P, Salo J, Jiménez $\mathrm{W}$, Inglada L, Navasa M, Claria J, Rimola A, Arroyo V, Rodés J: Incidence, predictive factors and prognosis of the hepatorenal syndrome. Gastroenterology 1993;105:229-236.

7 Zeller T, Bonvini RF, Sixt S: Color-coded duplex ultrasound for diagnosis of renal artery stenosis and as follow-up examination after revascularization. Catheter Cardiovasc Interv 2008;71:995-999.

-8 Chudek J, Kolonko A, Krol R, Ziaja J, Cierpka L, Wiecek A: The intrarenal vascular resistance parameters measured by duplex Doppler ultrasound shortly after kidney transplantation in patients with immediate, slow, and delayed graft function. Transplant Proc 2006;38:42-45.

-9 Wang Y, Liu L-P, Wen S-B, Dan H-J, Luan Y-Y, Zeng M-X, Hu B: Renal haemodynamics in patients with liver cirrhosis assessed by colour ultrasonography. J Int Med Res 2011; 39:249-255.
10 Götzberger M, Kaiser C, Landauer N, Dieterle C, Heldwein W, Schiemann U: Intrarenal resistance index for the assessment of early renal function impairment in patients with liver cirrhosis. Eur J Med Res 2008; 13: 383-387.

11 Sacerdoti A, Bolognesi M, Merkel C, Angeli $\mathrm{P}$, Gatta A: Renal vasoconstriction in cirrhosis evaluated by duplex Doppler ultrasonography. Hepatology 1993; 17: 219-224

- 12 Colli A, Cocciolo M, Riva C, Martinez E: Abnormal renovascular impedance in patients with hepatic cirrhosis: detection with duplex US. Radiologie1993;187:561-563.

13 Celebi H, Dönder E, Celiker H: Renal blood flow detection with Doppler Ultrasonography in patients with hepatic cirrhosis. Arch Intern Med 1997;157:564-567.

14 Conn HO, Leevy CM, Vlahcevic ZR, Rodgers JB, Maddrey WC, Seeff L, Levy LL: Comparison of lactulose and neomycin in the treatment of chronic portal-systemic encephalopathy. A double blind controlled trial. Gastroenterology 1977;72:573-583. 
15 Hanley JA, McNeil BJ: The meaning and use of the area under a receiver operating characteristic (ROC) curve. Radiology 1982;143: 29-36.

16 Hanley JA, McNeil BJ: A method of comparing the areas under receiver operating characteristic curves derived from the same cases. Radiology 1983;148:839-843.

-17 Kastelan S, Ljubicic N, Kastelan Z, Ostojic R, Uravic M: The role of duplex Doppler ultrasonography in the diagnosis of renal dysfunction and hepatorenal syndrome in patients with liver cirrhosis. Hepatogastroenterology 2004;51:1408-1412.
18 Platt J, Ellis J, Rubin J, Merion R, Lucey M: Renal duplex Doppler ultrasonography: a noninvasive predictor of kidney dysfunction and hepatorenal failure in liver disease. Hepatology 1994;20:362-369.

19 Berzigotti A, Casadei A, Magalotti D, Castaldini N, Losinno F, Rossi C, Zoli M: Renovascular impedance correlates with portal pressure in patients with liver cirrhosis. Radiology 2006;240:581-586.

20 Maroto A, Ginès A, Salo J, Claria J, Ginès P, Anibarro L, Jimenez W, Arroyo V, Rodes J: Diagnosis on functional kidney failure of cirrhosis with Doppler sonography: prognostic value of resistive index. Hepatology 1994;20:839-844.
21 Bardi A, Sapunar J, Oksenberg D, Poniachik J, Fernandez M, Paolinelli P, Orozco R, Biagini L: Intrarenal arterial Doppler ultrasonography in cirrhotic patients with ascites, with and without hepatorenal syndrome. Rev Med Chile 2002;130:173-180.

22 Gerbes AL, Gülberg V, Bilzer M, Vogeser M: Evaluation of serum cystatin C concentration as a marker of renal function in patients with cirrhosis of the liver. Gut 2002;50:106110 .

23 European Association for the Study of the Liver: EASL-EORTC Clinical Practice Guidelines: Management of hepatocellular carcinoma. J Hepatol 2012;56:908-943. 